University of Nebraska - Lincoln

DigitalCommons@University of Nebraska - Lincoln

Faculty Publications from the Department of Electrical \& Computer Engineering, Department Electrical and Computer Engineering

2010

\title{
Secure Communication over Fading Channels with Statistical QoS Constraints
}

Deli Qiao

University of Nebraska-Lincoln, dlqiao@ce.ecnu.edu.cn

M. Cenk Gursoy

University of Nebraska-Lincoln, gursoy@engr.unl.edu

Senem Velipasalar

University of Nebraska-Lincoln, velipasa@engr.unl.edu

Follow this and additional works at: https://digitalcommons.unl.edu/electricalengineeringfacpub

Part of the Electrical and Computer Engineering Commons

Qiao, Deli; Cenk Gursoy, M.; and Velipasalar, Senem, "Secure Communication over Fading Channels with Statistical QoS Constraints" (2010). Faculty Publications from the Department of Electrical and Computer Engineering. 128.

https://digitalcommons.unl.edu/electricalengineeringfacpub/128

This Article is brought to you for free and open access by the Electrical \& Computer Engineering, Department of at DigitalCommons@University of Nebraska - Lincoln. It has been accepted for inclusion in Faculty Publications from the Department of Electrical and Computer Engineering by an authorized administrator of DigitalCommons@University of Nebraska - Lincoln. 


\title{
Secure Communication over Fading Channels with Statistical QoS Constraints
}

\author{
Deli Qiao, Mustafa Cenk Gursoy, and Senem Velipasalar \\ Department of Electrical Engineering \\ University of Nebraska-Lincoln, Lincoln, NE 68588 \\ Email: dqiao726@huskers.unl.edu, gursoy@engr.unl.edu, velipasa@engr.unl.edu
}

\begin{abstract}
In this paper, secure transmission of information over an ergodic fading channel is studied in the presence of statistical quality of service $(\mathrm{QoS})$ constraints. We employ effective capacity to measure the secure throughput of the system, i.e., effective secure throughput. We assume that the channel side information (CSI) of the main and the eavesdropper channels is available at the transmitter side. Under this assumption, we investigate the optimal power control policies that maximize the effective secure throughput. In particular, it is noted that opportunistic transmission is no longer optimal and the transmitter should not wait to send the data at a high rate until the main channel is much better than the eavesdropper channel. Moreover, it is shown that the benefits of adapting the power with respect to the CSI of both the eavesdropper and main channels rather than the CSI of only the main channel diminish as QoS constraints become more stringent.
\end{abstract}

\section{INTRODUCTION}

Security is an important consideration in wireless systems due to the broadcast nature of wireless transmissions. In the pioneering work [1], Wyner addressed the security problem from an information-theoretic point of view and considered a wire-tap channel model. He proved that secure transmission of confidential messages to a destination in the presence of a degraded wire-tapper can be achieved, and he established the secrecy capacity which is defined as the highest rate of reliable communication from the transmitter to the legitimate receiver while keeping the wire-tapper completely ignorant of the transmitted messages. Recently, there has been numerous studies addressing information theoretic security. For instance, the impact of fading has been investigated in [2], where it has been shown that a non-zero secrecy capacity can be achieved even when the eavesdropper channel is better than the main channel on average. The secrecy capacity region of the fading broadcast channel with confidential messages and associated optimal power control policies have been identified in [3], where it is shown that the transmitter allocates more power as the strength of the main channel increases with respect to that of the eavesdropper channel.

In addition to security issues, providing acceptable performance and quality is vital to many applications. For instance, voice over IP (VoIP) and interactive-video (e.g,. videoconferencing) systems are required to satisfy certain buffer or

\footnotetext{
${ }^{1}$ This work was supported by the National Science Foundation under Grants CNS-0834753, and CCF-0917265.
}

delay constraints. In this paper, we consider statistical QoS constraints in the form of limitations on the buffer length, and incorporate the concept of effective capacity [4], which can be seen as the maximum constant arrival rate that a given timevarying service process can support while satisfying statistical QoS guarantees. The analysis and application of effective capacity in various settings have attracted much interest recently (see e.g., [5]-[7] and references therein). We define the effective secure throughput as the maximum constant arrival rate that can be supported while keeping the eavesdropper ignorant of these messages in the presence of QoS constraints. We assume that the channel side information (CSI) of the main and eavesdropper channels is available at the transmitter side. Under this assumption, we derive the optimal power control policies that maximize the effective secure throughput. We analyze two types of control policies by adapting the power with respect to both the main and eavesdropper channel conditions and also with respect to only the main channel conditions. Through this analysis, we find that, due to the introduction of the QoS constraints, the transmitter cannot reserve its power for times at which the main channel is much stronger than the eavesdropper channel. Also, we find that adapting the power allocation strategy with respect to both the main and eavesdropper channel CSI rather than only the main channel CSI provides little improvement when QoS constraints become more stringent.

The rest of the paper is organized as follows. Section II briefly describes the system model and the necessary preliminaries on statistical QoS constraints and effective capacity. In Section III, we present our results on power adaptation policies. Finally, Section IV concludes the paper.

\section{SyStem Model AND PRELIMINARIES}

\section{A. System Model}

The system model is shown in Fig. 1. It is assumed that the transmitter generates data sequences which are divided into frames of duration $T$. These data frames are initially stored in the buffer before they are transmitted over the wireless channel. The channel input-output relationships are given by

$$
\begin{aligned}
& Y_{1}[i]=h_{1}[i] X[i]+Z_{1}[i] \\
& Y_{2}[i]=h_{2}[i] X[i]+Z_{2}[i]
\end{aligned}
$$




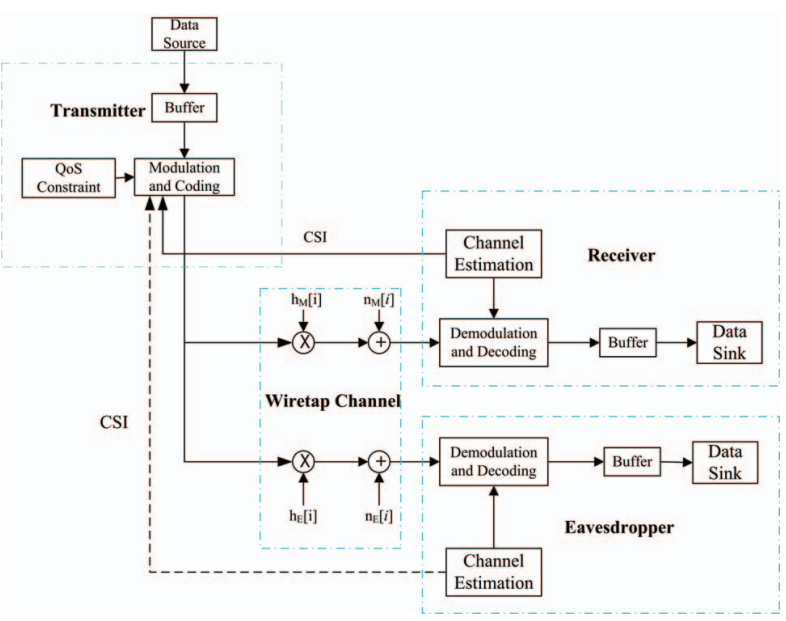

Fig. 1. The general system model.

where $i$ is the frame index, $X[i]$ is the channel input in the $i$ th frame, and $Y_{1}[i]$ and $Y_{2}[i]$ represent the channel outputs at receivers 1 and 2 in frame $i$, respectively. We assume that the sequences of fading coefficients $\left\{h_{j}[i]\right\}$ for $j=1,2$ are jointly stationary and ergodic discrete-time processes, and we denote the magnitude-square of the fading coefficients by $z_{j}[i]=\left|h_{j}[i]\right|^{2}$. Considering that receiver 1 is the main user and receiver 2 is the eavesdropper, we in the rest of the paper express $z_{1}$ and $z_{2}$ as $z_{M}$ and $z_{E}$, respectively, for more clarity. The channel input is subject to an average energy constraint $\mathbb{E}\left\{|X[i]|^{2}\right\} \leq \bar{P} / B$, where $B$ denotes the bandwidth available in the system. Assuming that there are $B$ complex symbols per second, we can easily see that the symbol energy constraint of $\bar{P} / B$ implies that the channel input has a power constraint of $\bar{P}$. Above in the channel inputoutput relationships, the noise component $Z_{j}[i]$ is a zero-mean, circularly symmetric, complex Gaussian random variable with variance $\mathbb{E}\left\{\left|Z_{j}[i]\right|^{2}\right\}=N_{j}$ for $j=1,2$. The additive Gaussian noise samples $\left\{Z_{j}[i]\right\}$ are assumed to form an independent and identically distributed (i.i.d.) sequence.

We denote the average transmitted signal to noise ratio with respect to receiver 1 as $\mathrm{SNR}=\frac{\bar{P}}{N_{1} B}$. We also denote $P[i]$ as the instantaneous transmit power in the $i$ th frame. Now, the instantaneous transmitted SNR level for receiver 1 can be expressed as $\mu^{1}[i]=\frac{P[i]}{N_{1} B}$. Then, the average energy constraint is equivalent to the average SNR constraint $\mathbb{E}\left\{\mu^{1}[i]\right\} \leq$ SNR for receiver 1 . If we denote the ratio between the noise power of the two channels as $\gamma=\frac{N_{1}}{N_{2}}$, the instantaneous transmitted SNR level for receiver 2 becomes $\mu^{2}[i]=\gamma \mu^{1}[i]$.

\section{B. Statistical QoS Constraints and Effective Secure Through- put}

In [4], Wu and Negi defined the effective capacity as the maximum constant arrival rate $^{2}$ that a given service process

\footnotetext{
${ }^{2}$ For time-varying arrival rates, effective capacity specifies the effective bandwidth of the arrival process that can be supported by the channel.
}

can support in order to guarantee a statistical QoS requirement specified by the QoS exponent $\theta$. If we define $Q$ as the stationary queue length, then $\theta$ is the decay rate of the tail of the distribution of the queue length $Q$ :

$$
\lim _{q \rightarrow \infty} \frac{\log P(Q \geq q)}{q}=-\theta .
$$

Therefore, for large $q_{\max }$, we have the following approximation for the buffer violation probability: $P\left(Q \geq q_{\max }\right) \approx$ $e^{-\theta q_{\max }}$. Hence, while larger $\theta$ corresponds to more strict QoS constraints, smaller $\theta$ implies looser QoS guarantees. Similarly, if $D$ denotes the steady-state delay experienced in the buffer, then $P\left(D \geq d_{\max }\right) \approx e^{-\theta \delta d_{\max }}$ for large $d_{\max }$, where $\delta$ is determined by the arrival and service processes [6].

The effective capacity is given by

$$
C(\theta)=-\frac{\Lambda(-\theta)}{\theta}=-\lim _{t \rightarrow \infty} \frac{1}{\theta t} \log _{e} \mathbb{E}\left\{e^{-\theta S[t]}\right\} \quad \text { bits/s, }
$$

where the expectation is with respect to $S[t]=\sum_{i=1}^{t} s[i]$, which is the time-accumulated service process. $\{s[i], i=$ $1,2, \ldots\}$ denotes the discrete-time stationary and ergodic stochastic service process. We define the effective capacity obtained when the service rate is confined by the secrecy capacity as the effective secure throughput.

In this paper, in order to simplify the analysis while considering general fading distributions, we assume that the fading coefficients stay constant over the frame duration $T$ and vary independently for each frame and each user. In this scenario, $s[i]=T R[i]$, where $R[i]$ is the instantaneous service rate for confidential messages in the $i$ th frame duration $[i T,(i+1) T]$. Then, (4) can be written as

$$
C(\theta)=-\frac{1}{\theta T} \log _{e} \mathbb{E}_{\mathbf{z}}\left\{e^{-\theta T R[i]}\right\} \quad \text { bits/s, }
$$

where $R[i]$ in general depends on the fading magnitudes $\mathbf{z}=$ $\left(z_{M}, z_{E}\right)$. (5) is obtained using the fact that instantaneous rates $\{R[i]\}$ vary independently over different frames. The effective secure throughput normalized by bandwidth $B$ is

$$
\mathrm{C}(\theta)=\frac{C(\theta)}{B} \quad \text { bits/s/Hz. }
$$

\section{SECRECy CAPACITY WITH QOS CONSTRAINTS}

In this section, we assume that the perfect CSI of the main and eavesdropper channels is available at the transmitter, and we analyze the performance in the presence of statistical QoS constraints. In particular, we study two types of power adaptation policies. First, we consider the case in which the power control policies take into account the CSI of both the main and eavesdropper channels. Subsequently, we investigate power allocation strategies that are functions of only the CSI of the main channel. 


\section{A. Power Adaptation with Main and Eavesdropper Channel State Information}

In this subsection, we assume that transmitter adapts the transmitted power according to the instantaneous values of $z_{M}$ and $z_{E}$ only when $z_{M}>\gamma z_{E}$. The secrecy capacity is then given by

$$
R_{s}= \begin{cases}B \log _{2}\left(1+\mu\left(z_{M}, z_{E}\right) z_{M}\right) & \\ -B \log _{2}\left(1+\gamma \mu\left(z_{M}, z_{E}\right) z_{E}\right), & z_{M}>\gamma z_{E} \\ 0, & \text { else. }\end{cases}
$$

where $\mu\left(z_{M}, z_{E}\right)$ is the optimal power allocated as a function of $z_{M}$ and $z_{E}$.

In the presence of QoS constraints, the optimal power allocation policy in general depends on the QoS exponent $\theta^{3}$. Hence, the secure throughput can be expressed as

$$
\begin{aligned}
& \mathrm{C}_{E}(\theta)=\max _{\substack{\mu\left(\theta, z_{M}, z_{E}\right) \\
\mathbb{E}\left\{\mu\left(\theta, z_{M}, z_{E}\right)\right\} \leq \mathrm{SNR}}}-\frac{1}{\theta T B} \log _{e}\left(\int_{0}^{\infty} \int_{0}^{\gamma z_{E}} p_{z_{M}}\left(z_{M}\right) p_{z_{E}}\left(z_{E}\right) d z_{M} d z_{E}\right. \\
& \left.\quad+\int_{0}^{\infty} \int_{\gamma z_{E}}^{\infty}\left(\frac{1+\mu\left(\theta, z_{M}, z_{E}\right) z_{M}}{1+\gamma \mu\left(\theta, z_{M}, z_{E}\right) z_{E}}\right)^{-\beta} p_{z_{M}}\left(z_{M}\right) p_{z_{E}}\left(z_{E}\right) d z_{M} d z_{E}\right)
\end{aligned}
$$

where $p_{z_{M}}\left(z_{M}\right)$ and $p_{z_{E}}\left(z_{E}\right)$ are the probability density functions of $z_{M}$ and $z_{E}$, respectively, and $\beta=\frac{\theta T B}{\log _{e} 2}$. Note that the first term in the $\log$ function is a constant and $\log$ is a monotonically increasing function. Therefore, the maximization problem in (8) is equivalent to the following minimization problem

$$
\begin{aligned}
\min _{\substack{\mu\left(\theta, z_{M}, z_{E}\right) \\
\mathbb{E}\left\{\mu\left(\theta, z_{M}, z_{E}\right)\right\} \leq \mathrm{SNR}}} & \int_{0}^{\infty} \int_{\gamma z_{E}}^{\infty}\left(\frac{1+\mu\left(\theta, z_{M}, z_{E}\right) z_{M}}{1+\gamma \mu\left(\theta, z_{M}, z_{E}\right) z_{E}}\right)^{-\beta} \\
& \times p_{z_{M}}\left(z_{M}\right) p_{z_{E}}\left(z_{E}\right) d z_{M} d z_{E}
\end{aligned}
$$

It is easy to check that when $z_{M}>\gamma z_{E}$,

$$
f(\mu)=\left(\frac{1+\mu z_{M}}{1+\gamma \mu z_{E}}\right)^{-\beta}
$$

is a convex function in $\mu$. Since nonnegative weighted sum of convex functions is convex [9], we can immediately see that the objective function in (9) is also convex in $\mu$. Then, we can form the following Lagrangian function, denoted as $\mathcal{J}$ :

$\begin{aligned} \mathcal{J} & =\int_{0}^{\infty} \int_{\gamma z_{E}}^{\infty}\left(\frac{1+\mu\left(\theta, z_{M}, z_{E}\right) z_{M}}{1+\gamma \mu\left(\theta, z_{M}, z_{E}\right) z_{E}}\right)^{-\beta} p_{z_{M}}\left(z_{M}\right) p_{z_{E}}\left(z_{E}\right) d z_{M} d z^{\infty} \\ & +\lambda\left(\int_{0}^{\infty} \int_{\gamma z_{E}}^{\infty} \mu\left(\theta, z_{M}, z_{E}\right) p_{z_{M}}\left(z_{M}\right) p_{z_{E}}\left(z_{E}\right) d z_{M} d z_{E}-\mathrm{SNR}\right)\end{aligned}$

Taking the derivative of the Lagrangian function over

\footnotetext{
${ }^{3}$ Due to this dependence, we henceforth use $\mu\left(\theta, z_{M}, z_{E}\right)$ to denote the power allocation policy under QoS constraints.
}

$\mu\left(\theta, z_{M}, z_{E}\right)$, we get the following optimality condition:

$$
\begin{aligned}
& \frac{\partial \mathcal{J}}{\partial \mu\left(\theta, z_{M}, z_{E}\right)}=\lambda-\beta\left(\frac{1+\mu\left(\theta, z_{M}, z_{E}\right) z_{M}}{1+\gamma \mu\left(\theta, z_{M}, z_{E}\right) z_{E}}\right)^{-\beta} \\
& \quad \times \frac{z_{M}-\gamma z_{E}}{\left(1+\mu\left(\theta, z_{M}, z_{E}\right) z_{M}\right)\left(1+\gamma \mu\left(\theta, z_{M}, z_{E}\right) z_{E}\right)}=0
\end{aligned}
$$

where $\lambda$ is the Lagrange multiplier whose value is chosen to satisfy the average power constraint with equality. For any channel state pairs $\left(z_{M}, z_{E}\right), \mu\left(\theta, z_{M}, z_{E}\right)$ can be obtained from the above condition. Whenever the value of $\mu\left(\theta, z_{M}, z_{E}\right)$ is negative, it follows from the convexity of the objective function with respect to $\mu\left(\theta, z_{M}, z_{E}\right)$ that the optimal value of $\mu\left(\theta, z_{M}, z_{E}\right)$ is 0 .

There is no closed-form solution to (12). However, since the right-hand side (RHS) of (12) is a monotonically increasing function, numerical techniques such as bisection search method can be efficiently adopted to derive the solution.

The secure throughput can be determined by substituting the optimal power control policy $\mu^{*}\left(\theta, z_{M}, z_{E}\right)$ in (8). Exploiting the optimality condition in (12), we can notice that when $\mu\left(\theta, z_{M}, z_{E}\right)=0$, we have $z_{M}-\gamma z_{E}=\frac{\lambda}{\beta}$. Meanwhile,

$$
\begin{aligned}
& \left(\frac{1+\mu\left(\theta, z_{M}, z_{E}\right) z_{M}}{1+\gamma \mu\left(\theta, z_{M}, z_{E}\right) z_{E}}\right)^{-\beta} \\
& \times \frac{1}{\left(1+\mu\left(\theta, z_{M}, z_{E}\right) z_{M}\right)\left(1+\gamma \mu\left(\theta, z_{M}, z_{E}\right) z_{E}\right)}<1 .
\end{aligned}
$$

Thus, we must have $z_{M}-\gamma z_{E}>\frac{\lambda}{\beta}$ for $\mu\left(\theta, z_{M}, z_{E}\right)>0$, i.e., $\mu\left(\theta, z_{M}, z_{E}\right)=0$ if $z_{M}-\gamma z_{E} \leq \frac{\lambda}{\beta}$. Hence, we can write the secure throughput as

$$
\begin{aligned}
C_{E}(\theta)=-\frac{1}{\theta T B} \log _{e}\left(\int_{0}^{\infty} \int_{0}^{\gamma z_{E}+\frac{\lambda}{\beta}} p_{z_{M}}\left(z_{M}\right) p_{z_{E}}\left(z_{E}\right) d z_{M} d z_{E}\right. \\
+\int_{0}^{\infty} \int_{\gamma z_{E}+\frac{\lambda}{\beta}}^{\infty}\left(\frac{1+\mu^{*}\left(\theta, z_{M}, z_{E}\right) z_{M}}{1+\gamma \mu^{*}\left(\theta, z_{M}, z_{E}\right) z_{E}}\right)^{-\beta} \\
\left.\times p_{z_{M}}\left(z_{M}\right) p_{z_{E}}\left(z_{E}\right) d z_{M} d z_{E}\right)
\end{aligned}
$$

where $\mu^{*}\left(\theta, z_{M}, z_{E}\right)$ is the derived optimal power control policy.

B. Power Adaptation with only Main Channel State Information

In this section, we assume that the transmitter adapts the power level by only taking into account the CSI of the main channel (the channel between the transmitter and the legitimate receiver). Under this assumption, the secrecy rate for a specific channel state pair becomes

$$
R_{s}=\left[B \log _{2}\left(1+\mu\left(z_{M}\right) z_{M}\right)-B \log _{2}\left(1+\gamma \mu\left(z_{M}\right) z_{E}\right)\right]^{+}
$$

where $\mu\left(z_{M}\right)$ is the optimal power allocated as a function of only $z_{M}$. 


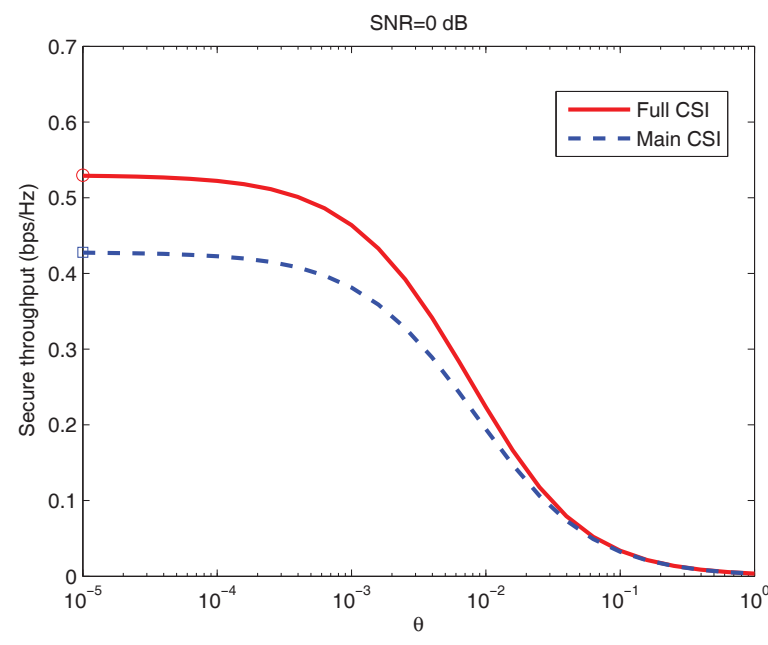

Fig. 2. The effective secure throughput vs. $\theta$ in the Rayleigh fading channel with $\mathbb{E}\left\{z_{E}\right\}=\mathbb{E}\left\{z_{M}\right\}=1 . \gamma=1$.

In this case, the secure throughput can be expressed as

$$
\begin{aligned}
& \mu\left(\theta, z_{M}, z_{E}\right)=0, \text { we have } \\
& -\beta \int_{0}^{z_{M} / \gamma}\left(z_{M}-\gamma z_{E}\right) p_{z_{E}}\left(z_{E}\right) d z_{E}+\lambda=0 \\
& \Rightarrow \int_{0}^{z_{M}} P\left(z_{E} \leq t / \gamma\right) d t=\frac{\lambda}{\beta}
\end{aligned}
$$

Let us denote the solution to the above equation as $\alpha$. Considering that

$$
\left(\frac{1+\mu\left(\theta, z_{M}\right) z_{M}}{1+\gamma \mu\left(\theta, z_{M}\right) z_{E}}\right)^{-\beta-1} \frac{1}{\left(1+\gamma \mu\left(\theta, z_{M}\right) z_{E}\right)^{2}}<1,
$$

we must have $z_{M}>\alpha$ for $\mu\left(\theta, z_{M}\right)>0$, i.e., $\mu\left(\theta, z_{M}\right)=0$ if $z_{M} \leq \alpha$. Hence, we can write the secure throughput as

$$
\begin{aligned}
C_{E}(\theta)= & -\frac{1}{\theta T B} \log _{e}\left(\int_{0}^{\alpha} \int_{0}^{\infty} p_{z_{M}}\left(z_{M}\right) p_{z_{E}}\left(z_{E}\right) d z_{E} d z_{M}\right. \\
& +\int_{\alpha}^{\infty} \int_{z_{M} / \gamma}^{\infty} p_{z_{M}}\left(z_{M}\right) p_{z_{E}}\left(z_{E}\right) d z_{E} d z_{M} \\
+ & \int_{\alpha}^{\infty} \int_{0}^{z_{M} / \gamma}\left(\frac{1+\mu^{*}\left(\theta, z_{M}\right) z_{M}}{1+\gamma \mu^{*}\left(\theta, z_{M}\right) z_{E}}\right)^{-\beta} \\
& \left.\times p_{z_{M}}\left(z_{M}\right) p_{z_{E}}\left(z_{E}\right) d z_{E} d z_{M}\right)
\end{aligned}
$$

where $\mu^{*}\left(\theta, z_{M}\right)$ is the derived optimal power control policy.

C. Numerical Results

Similar to the discussion in Section III-A, we get the following equivalent minimization problem:

$$
\begin{aligned}
\min _{\substack{\mu\left(\theta, z_{M}\right) \\
\mathbb{E}\left\{\mu\left(\theta, z_{M}\right)\right\} \leq \mathrm{SNR}}} \int_{0}^{\infty} \int_{0}^{z_{M} / \gamma} & \left(\frac{1+\mu\left(\theta, z_{M}\right) z_{M}}{1+\gamma \mu\left(\theta, z_{M}\right) z_{E}}\right)^{-\beta} \\
& \times p_{z_{M}}\left(z_{M}\right) p_{z_{E}}\left(z_{E}\right) d z_{E} d z_{M}
\end{aligned}
$$

The objective function in this case is again convex, and with a similar Lagrangian optimization method, we can get the following optimality condition:

$$
\begin{aligned}
\frac{\partial \mathcal{J}}{\partial \mu\left(\theta, z_{M}\right)}=- & \beta \int_{0}^{z_{M} / \gamma}\left(\frac{1+\mu\left(\theta, z_{M}\right) z_{M}}{1+\gamma \mu\left(\theta, z_{M}\right) z_{E}}\right)^{-\beta-1} \\
& \times \frac{z_{M}-\gamma z_{E}}{\left(1+\gamma \mu\left(\theta, z_{M}\right) z_{E}\right)^{2}} p_{z_{E}}\left(z_{E}\right) d z_{E}+\lambda=0
\end{aligned}
$$

In Fig. 2, we plot the effective secure throughput as a function of the QoS exponent $\theta$ in Rayleigh fading channel with $\gamma=1$ when the power is adapted with respect to the full CSI (i.e., the CSI of main and eavesdropper channels) and also with respect to only the main CSI. It can be seen from the figure that as the QoS constraints become more stringent and hence as the value of $\theta$ increases, little improvement is provided by considering the CSI of the eavesdropper channel in the power adaptation. In Fig. 3, we plot the effective secure throughput as SNR varies for $\theta=\{0,0.001,0.01,0.1\}$. Not surprisingly, we again observe that taking into account the CSI of the eavesdropper channel in the power adaptation policy does not provide much gains in terms of increasing the effective secure throughput in the large SNR regime. Also, as QoS constraints become more strict, we similarly note that adapting the power with full CSI does not increase the rate of secure transmission much even at medium SNR levels.

To characterize the power allocation strategy, we plot the power distribution as a function of $\left(z_{E}, z_{M}\right)$ for the full CSI case when $\theta=0.01$ and $\theta=0$ in Fig. 4. In the figure, we see that for both values of $\theta$, no power is allocated for transmission when $z_{M}<z_{E}$ which is expected under the assumption of equal noise powers, i.e., $N_{1}=N_{2}$. We note that when $\theta=0$ and hence there are no buffer constraints, opportunistic transmission policy is employed. More power is allocated for cases in which the difference $z_{M}-z_{E}$ is large. Therefore, the transmitter favors the times at which the main channel is much better than the eavesdropper channel. At these times, 


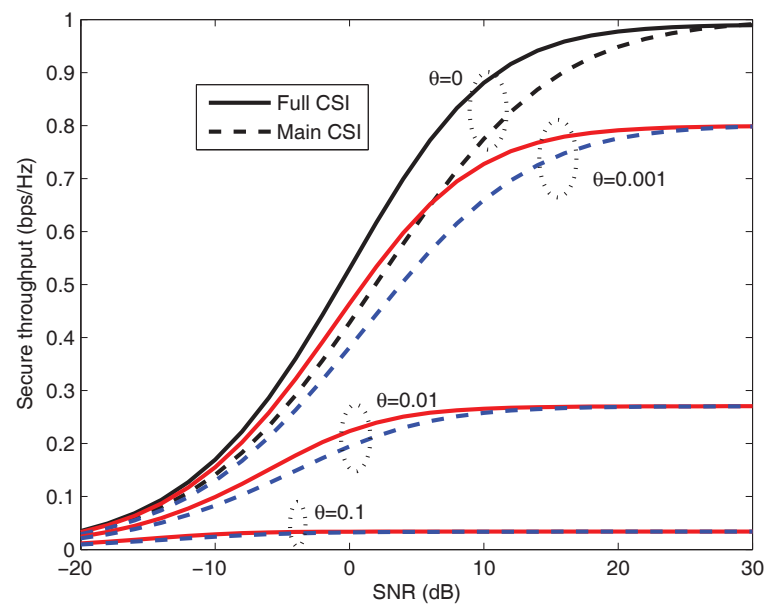

Fig. 3. The effective secure throughput vs. SNR in the Rayleigh fading channel with $\mathbb{E}\left\{z_{E}\right\}=\mathbb{E}\left\{z_{M}\right\}=1$. $\gamma=1$.

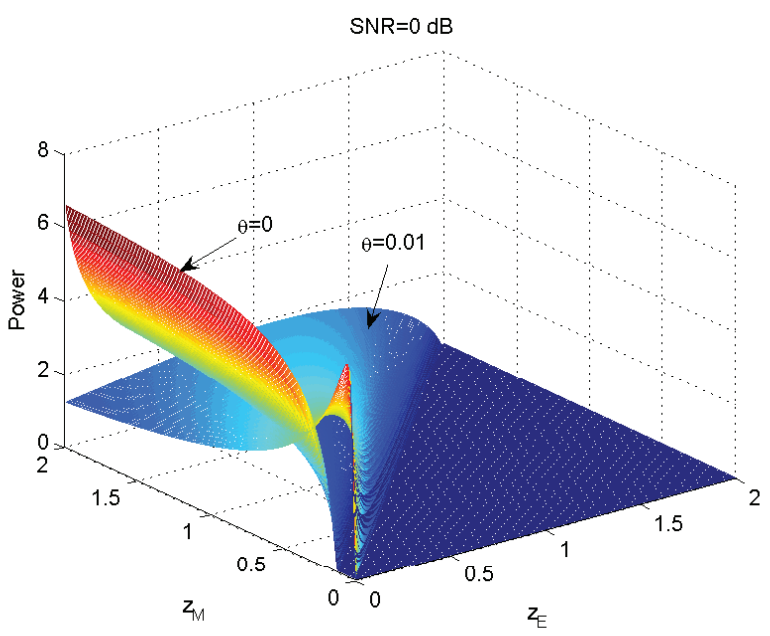

Fig. 4. The power allocation for the full CSI scenario with $\mathrm{SNR}=0 \mathrm{~dB}$ in the Rayleigh fading channel with $\mathbb{E}\left\{z_{E}\right\}=\mathbb{E}\left\{z_{M}\right\}=1 . \gamma=1$.

the transmitter sends the information at a high rate with large power. When $z_{M}-z_{E}$ is small, transmission occurs at a small rate with small power. However, this strategy is clearly not optimal in the presence of buffer constraints because waiting to transmit at a high rate until the main channel becomes much stronger than the eavesdropper channel can lead to buildup in the buffer and incur large delays. Hence, we do not observe this opportunistic transmission strategy when $\theta=0.01$. In this case, we note that a more uniform power allocation is preferred. In order not to violate the limitations on the buffer length, transmission at a moderate power level is performed even when $z_{M}-z_{E}$ is small.

\section{CONCLUSiON}

In this paper, we have analyzed the secrecy capacity in the presence of statistical QoS constraints. We have considered the effective secure throughput as a measure of the performance. With different assumptions on the use of the main and eavesdropper CSI in the power control strategies, we have investigated the associated optimal power allocation policies that maximize the effective secure throughput. In particular, we have noted that the transmitter allocates power more uniformly instead of concentrating its power for the cases in which the main channel is much stronger than the eavesdropper channel. By numerically comparing the obtained effective secure throughput, we have shown that as QoS constraints become more stringent, the benefits of incorporating the CSI of the eavesdropper channel in the power control policy diminish.

\section{REFERENCES}

[1] A. D. Wyner, "The wire-tap channel," Bell Syst. Tech. J., vol. 54, pp. 1355-1387, Oct. 1975.

[2] P. Gopala, L. Lai, and H. El Gamal, "On the secrecy capacity of fading channels," IEEE Trans. Inform. Theo., vol. 54, no. 10, pp. 4687-4698, Oct. 2008.

[3] Y. Liang, H. V. Poor, and S. Shamai, "Secure communication over fading channels,"IEEE Trans. Inform. Theory, vol. 54, pp. 2470-2492, June 2008.

[4] D. Wu and R. Negi "Effective capacity: a wireless link model for support of quality of service," IEEE Trans. Wireless Commun., vol.2,no. 4, pp.630-643. July 2003

[5] J. Tang and X. Zhang, "Quality-of-service driven power and rate adaptation over wireless links," IEEE Trans. Wireless Commun., vol. 6, no. 8, pp. 3058-3068, Aug. 2007.

[6] J. Tang and X. Zhang, "Cross-layer-model based adaptive resource allocation for statistical QoS guarantees in mobile wireless networks," IEEE Trans. Wireless Commun., vol. 7, no. 6, pp.2318-2328, June 2008.

[7] L. Liu, P. Parag, and J.-F. Chamberland, "Quality of service analysis for wireless user-cooperation networks," IEEE Trans. Inform. Theory, vol. 53, no. 10 , pp. $3833-3842$, Oct. 2007

[8] A. Goldsmith, Wireless Communications, 1st ed. Cambridge University Press, 2005.

[9] S. Boyd and L. Vandenberghe, Convex Optimization, Cambridge University Press, 2004. 